\title{
Dicrotic Pulse Revisited in the Pandemic Context
}

\author{
Bhushan Chavan ${ }^{1} \cdot$ Raghavannair Suresh Kumar² ${ }^{\circledR}$
}

Received: 21 July 2021 / Accepted: 2 August 2021 / Published online: 8 August 2021

(c) The Author(s), under exclusive licence to Springer Science+Business Media, LLC, part of Springer Nature 2021

In 1902, Mackenzie described the dicrotic pulse as an abnormal central pulse seen in typhoid fever, where there were two palpable pulsations [1]. Wood found it a good sign of vascular relaxation in patients with infectious or toxic conditions [2]. The COVID pandemic has given us reason to revisit this clinical sign.

The dicrotic notch is the nadir point that occurs immediately after the closure of the aortic valve marking the beginning of the dicrotic wave [3]. Dicrotic notch is usually seen one-third of the way down the descending limb of the pressure wave (Fig. 1a). The secondary dicrotic wave occurs as the elastic recoil of the arterial tree clamps down against a closed aortic valve causing a secondary pressure increase [3]. The aortic valve closure occurs when aortic pressure exceeds the left ventricular pressure. In presence of marked vasodilatation, this pressure gradient occurs later in the cardiac cycle, pushing the dicrotic notch down [3]. The resultant dicrotic pulse has a low systolic pressure, low diastolic pressure, and widened pulse pressure. The single systolic wave, low dicrotic notch, and the large dicrotic wave produce a characteristic "M" pattern [3] (Fig. 1b).

The COVID-19 pandemic has brought in its train a new syndrome which has been called MIS-C in the USA [4].
This may present with multi-organ failure and cardiogenic or vasoplegic shock requiring inotropic support $[4,5]$. Vasoplegic shock would need vasopressors which may be detrimental in cardiogenic shock. Vasoplegic shock is the typical situation where dicrotic pulse occurs. Feinberg and Lax demonstrated marked difference between the effects of epinephrine and norepinephrine on the dicrotic wave-the former exaggerating and the latter abolishing it-showing the role of the arterial tone in generating the distinctive wave pattern [6] and giving us a clue to the management of vasoplegic shock.

A thirteen-year-old boy presented to us with high-grade fever and raised inflammatory markers suggestive of MIS-C. The arterial pressure trace and the SPO2 pulse trace (supplementary material A) clearly showed a dicrotic pulse. Few hours after recording this, the child went into hypotension and was revived with vasopressin infusion and fluids. The pulse trace now normalized with the dicrotic notch moving upward (supplementary material B).

The current report suggests that demonstration of a dicrotic pulse would suggest vasoplegia as the cause of shock in MIS-C. Early institution of vasoconstrictor therapy is likely to prevent clinical deterioration in such cases.

Raghavannair Suresh Kumar

r.sureshkumar.mmm@gmail.com

1 Apollo Hospital, Navi Mumbai, India

2 Believers International Heart Center, Thiruvalla, Kerala, India 


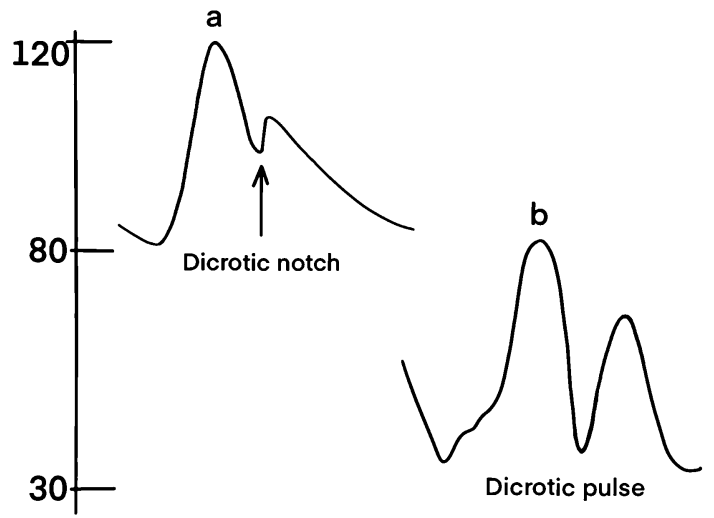

Fig. 1 a Normal pulse wave, b Dicrotic pulse wave

Supplementary Information The online version contains supplementary material available at https://doi.org/10.1007/s00246-021-02702-1.

\section{Declarations}

Conflict of interest The authors declare that there are no conflicts of interest in the case reported.

Informed Consent There is informed consent from the parents for publishing the data of the case reported. The report does not involve research on humans or animals.

\section{References}

1. Smith D, Craige E (1986) Mechanism of the dicrotic pulse. $\mathrm{Br}$ Heart J 56:531-534

2. Wood P (1956) Diseases of the heart and circulation. J. B. Lippincott Co., Philadelphia, pp 28, 610

3. Nirmalan M, Dark PM (2014) Broader applications of arterial pressure wave form analysis. Continuing Education in Anaesthesia. Crit Care Pain 14(6):285-290

4. Sperotto F, Friedman KG, Son MBF, VanderPluym CJ et al (2021) Cardiac manifestations in SARS-CoV-2-associated multisystem inflammatory syndrome in children: a comprehensive review and proposed clinical approach. Eur J Pediatr 180:307-322

5. Ramcharan T, Nolan O, Lai CY et al (2020) Paediatric inflammatory multisystem syndrome: temporally associated with SARSCoV-2 (PIMS-TS): cardiac features, management and short-term outcomes at a UK Tertiary Paediatric Hospital. Pediatr Cardiol. https://doi.org/10.1007/s00246-020-02391-2

6. Feinberg AW, Lax H (1958) Studies of the arterial pulse wave. Circulation 18:1125

Publisher's Note Springer Nature remains neutral with regard to jurisdictional claims in published maps and institutional affiliations. 